\title{
FOTO

\section{El sueño espacial europeo como alternativa en Próxima, de Alice Winocour (2019)}

\section{The European space dream as an alternative in Alice Winocour's Proxima (2019)}

\author{
Antonio Sánchez-Escalonilla \\ Universidad Rey Juan Carlos (Madrid) \\ antonio.sanchezescalonilla@urjc.es
}

\section{Resumen:}

El estreno del filme Próxima en 2019, realizado por la directora francesa Alice Winocour, ha establecido un hito en el género cinematográfico de viajes espaciales al tratarse de la primera producción protagonizada por una mujer astronauta europea, madre de familia, que realiza su sueño de viajar a las estrellas en una misión de la Estación Espacial Internacional. El contraste de la protagonista con sus compañeros de tripulación -un cosmonauta ruso de Roscosmos y un astronauta estadounidense de la NASA-, da lugar una confrontación de diversos imaginarios: espacial, geopolítico y de roles femeninos y masculinos. El presente artículo pretende analizar esta confrontación socionarrativa presente en Próxima, tomando como referente el sueño espacial europeo en cuanto ethos alternativo al sueño americano y al sueño ruso/soviético.

\begin{abstract}
:
The premiere of the film Proxima in 2019 by French filmmaker Alice Winocour established a milestone in the space travel genre by being the first featuring a European woman astronaut as the main protagonist, a family mother whose dream of joining a mission on the International Space Station come true. Conflicts with her crewmates -a Roscosmos cosmonaut and a NASA astronaut- lead to a clash of multiple world views that encompass space, geopolitics and gender roles. This article aims to analyze this socionarrative frame in Winocour's film, by looking at the European space dream as both a reference and an alternative ethos to the American dream and the Russian/Soviet dream.
\end{abstract}

Palabras clave: Sueño europeo; cine de exploración espacial; mujer astronauta; maternidad; Alice Winocour; imaginarios fílmicos.

Keywords: European dream; Space exploration cinema; Woman astronaut; Motherhood; Alice Winocour; Film imaginaries. 


\section{Introducción ${ }^{*}$}

Desde los primeros años de la Guerra Fría, Estados Unidos y la Unión Soviética plantearon la exploración espacial en términos geopolíticos, como una extensión de sus respectivas estrategias de control militar y desarrollo armamentista. Al mismo tiempo, la conquista tecnológica del espacio se expresaba en los medios y en las artes como una narrativa patriótica que encontró en el cine un eficaz instrumento divulgador. Así, a finales de los años 50 el sueño espacial pasó a formar parte de los imaginarios del American Dream (McCurdy 2011, Allen 2009) y del ethos soviético (Spufford 2010, Smith 2014). Avanzada la carrera espacial, los progresos del programa soviético y de la NASA favorecieron el desarrollo del género cinematográfico de viajes espaciales, estableciendo en la audiencia un fenómeno de realimentación entre ficción y tecnología que McDougall (1985) denomina "anticipación cultural".

Entre tanto, los ciudadanos europeos contemplaban la carrera espacial como meros espectadores, divididos por un telón de acero que les obligaba a apoyar en la distancia los hitos alcanzados desde el cosmódromo de Baikonur y Cabo Cañaveral. Entre los años 50 y 70 no era posible hablar de un sueño espacial europeo, alternativo o "no alineado", comparable al soviético o al estadounidense, más allá de la colaboración que los países satélites mantenían con las agencias de cada potencia. Durante décadas, este factor obligó a las audiencias europeas a nutrir su fantasía con una iconografía fílmica ajena a su identidad continental (Collins 1990). Los programas no tripulados de la Agencia Espacial Europea (AEE), surgida en 1975 gracias al impulso de diez países fundadores, resultaron insuficientes para alimentar el imaginario de un sueño autóctono (Abeelen 2016). Por otro lado, la participación de astronautas europeos en los programas de la Estación Espacial Internacional (EEI), junto a tripulantes rusos y estadounidenses, siempre presentaba un carácter secundario. Como consecuencia, la ausencia de un ethos y de una iconografía espacial, unida a la inexistencia de

\footnotetext{
* Artículo resultado del proyecto I+D+i "La crisis del European Dream: hogar, identidad y éxodo en las artes audiovisuales" financiado por el MINECO, convocatoria del Programa Retos de la Sociedad 2017, con referencia HAR2017-85846-R.
} 
referentes populares, explica en buena medida la carencia de una filmografía europea sobre misiones de exploración espacial.

Dado este contexto histórico y socionarrativo previo, el estreno del filme Próxima en 2019, realizado por Alice Winocour, ha establecido un hito en el género de viajes espaciales. Se trata de una producción franco-alemana, protagonizada por una astronauta francesa de la AEE y dirigido por una directora también de origen francés, que deseaba narrar la aventura de una madre que se entrena para volar hasta la órbita terrestre. Más allá de la significancia que supone este título en la historia del cine europeo, el filme encierra una propuesta de reflexión sobre la realidad de un sueño espacial alternativo, tras varias décadas de predominio de la polaridad rusoestadounidense en el imaginario espacial. Al mismo tiempo, Próxima plantea en términos sociales una tercera vía entre el American dream y el sueño soviético, hoy día reconvertido en un ethos ruso anclado en la nostalgia por la Guerra Fría. Esta opción intermedia apunta a un concepto de prosperidad social basado en los vínculos personales y transnacionales, lejos de tensiones geopolíticas, donde la figura femenina ocupa un papel primordial. A este respecto, Winocour explica:

Sarah es superheroína y madre en un único cuerpo. El cine no suele mostrar estos dos aspectos en una única persona, como si fuera incompatible ser heroína y ser madre. Las superheroínas siempre se nos muestran separadas de cuestiones como la maternidad o la feminidad en la vida diaria. Ese es el aspecto feminista de la película: mostrar que una mujer puede ser madre y a la vez una profesional de altos vuelos (2019, p. 3).

Próxima aparece al término de una década oscura para Europa, marcada por la crisis, el declive social y el atrincheramiento detrás de sus fronteras ${ }^{1}$, aunque paradójicamente animada por un florecimiento cultural. Así lo entienden Harrod, Liz y Timoshkina cuando aseguran que, en nuestros días,

\footnotetext{
${ }^{1}$ En 2015 se registraron 3.771 víctimas de migrantes que intentaban cruzar las fronteras marítimas europeas (Migration Data Portal 2015). Según un informe del Council for Foreign Relations, "pese al alto número de víctimas, la respuesta colectiva de los países de la Unión Europea al flujo migratorio ha sido coyuntural, y más preocupada en asegurar las fronteras del bloque comunitario que en proteger los derechos de migrantes y refugiados" (Park, 2015).
} 
"Europa es más visible que nunca, pero su imagen es asimismo más negativa que nunca” (2015, p. 5). A través de un sueño espacial emergente, transversal en el ámbito político y personalista en el social, la directora considera su filme como una apuesta por la exploración espacial como base de la cooperación internacional: "La dimensión internacional se veía reflejada en la composición de nuestro equipo, que incluía a franceses, rusos, estadounidenses, alemanes y kazajos. Esa mezcla de nacionalidades nos hacía sentir unidos en nuestra humanidad compartida (2019, p. 4).

\section{Marco teórico y Metodología}

En su filme, Winocour establece un vínculo entre progreso y realización de un sueño particular, mientras explora las dificultades de la mujer a la hora de afrontar retos profesionales y vitales en igualdad de condiciones. En este sentido, el contraste de la protagonista con sus compañeros de tripulación un cosmonauta ruso de Roscosmos y un astronauta estadounidense de la NASA-, da lugar una interesante confrontación que abarca diversos imaginarios: el espacial, el socionarrativo, el geopolítico y el de los roles femeninos y masculinos. El presente artículo pretende analizar esta confrontación de imaginarios narrativos presentes en Próxima, tomando como referente el sueño espacial europeo en cuanto ethos alternativo.

Para abordar este análisis, partimos de la dualidad polarizada en los años de la Guerra Fría, momento en que se consolidan el imaginario espacial y los referentes tradicionales del género. Sobre esta base, el impacto de Próxima puede calibrarse no solo en cuanto hito para la narrativa de los sueños espacial y europeo, sino también como una muestra de la renovación iconográfica del género cinematográfico y del imaginario de la heroína femenina, tanto en tierra como en el cosmos.

El reciente concepto de sueño europeo se ha entendido como la persecución de un futuro próspero para las naciones de Europa, concebida como unidad en una identidad democrática supranacional. Según Rifkin, el European Dream prometía "llevar la humanidad a una conciencia global, más ajustada 
a una sociedad crecientemente interconectada y globalizada” (2004, p. 3), planteamiento que de entrada diverge de los ethos previos estadounidense y soviético, el primero basado en la autonomía para el éxito individual (Samuel 2012), y el segundo en la difuminación del individuo como requisito para la prosperidad colectiva (Spufford 2010). Para Rifkin, el sueño europeo basa la felicidad de sus ciudadanos en dos conceptos: el acceso al progreso por parte de otras comunidades y la garantía a opciones de vida plena. Estos criterios se tendrán como referencia en el análisis de Próxima, a través de los contrastes de los tres imaginarios representados en la tripulación del cohete Soyuz.

\section{Consolidación de los imaginarios espaciales estadounidense y ruso}

Como se ha comentado atrás, los medios de comunicación y el cine en particular promovieron durante la carrera espacial una iconografía de carácter nacionalista, de modo que el sueño espacial se integró en los imaginarios opuestos del American Dream y del ethos soviético. En efecto, si Pravda consideraba el lanzamiento del Sputnik en 1957 como "una gran victoria en la competición global contra el capitalismo" que hizo posible "los deseos de generaciones de soñadores espaciales" (Andrews y Saddiqi, 2011, p. 5), Kennedy asociaba su reto de alcanzar la Luna antes de 1970 con la conquista de nuevos conocimientos y derechos², en sintonía con las tesis pioneras de la frontera (Turner 2014) y la definición de sueño americano acuñada en 1931 por Adams33.

Este vínculo entre sueño espacial e identidad nacional ya había sido preconizado en las artes durante el período de entreguerras. Así, Smirnovna vincula las vanguardias rusas de los años 20 con un entusiasmo popular por

\footnotetext{
2 Discurso en la Universidad de Rice, Houston. John F. Kennedy: 12 de septiembre de 1962. 3 "Un sueño de orden social en el que cada hombre y cada mujer están en condiciones de alcanzar la más alta aspiración de la que son capaces por naturaleza, y de ser reconocidos por lo que son, con independencia de las circunstancias fortuitas de su nacimiento y posición" (Adams, 1931, p. 215).
} 
el desarrollo tecnológico y la ciencia-ficción: “Aquellos artistas no solo deseaban representar el mundo: querían transformarlo. Yuon, que pintó su obra a comienzos de los años 20, mientras la guerra civil todavía rugía en Rusia, retrata la revolución como el nacimiento de un nuevo planeta” (House 2016). En la década siguiente, asentada la revolución, el sueño espacial ruso se vio impulsado entre las audiencias gracias al filme El viaje cósmico (Kosmicheskii Reis, 1936), del director Vasily Zhuravlyov, que relataba la primera expedición tripulada a la Luna. A bordo de la nave Josef Stalin, el viejo profesor Sedikh aterriza sobre el satélite en 1946, acompañado por sus ayudantes Marina y Andryusha, y consigue regresar con éxito a la Tierra: "la película combinaba el paradigma ideológico social-realista, de cuño estalinista, con los sueños y esperanzas creativos, menos politizados, del anciano personaje de la vida real K. E. Tsiolkovski (Andrews, 2011, p. 38).

Por otro lado, las ilustraciones del artista Chesley Bonestell, publicadas en la revista Life a comienzos de los años 40, provocaron entre el público estadounidense un fenómeno equivalente al de las pinturas de Yuon en el ruso. La serie de ilustraciones planetarias realizadas por Bonestell para Life, se convirtieron en uno de los iconos emblemáticos del sueño espacial colectivo, gracias a la evocación del espíritu de la frontera en los panoramas celestes. Así lo aprecia Wachhorst en The Dream of the Spaceflight:

El hallazgo de los cielos azules de Titán en Bonestell, el encuentro con el rojizo desierto rocoso de Marte resultan tan cautivadores como el suroeste de Estados Unidos, y sugiere que el límite más cercano del universo es, en el fondo, una realidad tan familiar como el patio trasero de nuestra propia casa (2000, pp. 57-58).

Iniciada la carrera espacial, el imaginario estadounidense de los años 60 y 70 quedó marcado en esta etapa por dos títulos capitales: 2001: una odisea del espacio (2001: A Space Odyssey, Stanley Kubrick 1968) y Atrapados en el espacio (Marooned, John Sturges 1969). 2001 ofrecía la imagen de un futuro a treinta años vista en el que se han cumplido todas las etapas del sueño espacial, de acuerdo con las previsiones de científicos como Ley, Whipple, y Von Braun: establecimiento de estaciones orbitales en torno a la Tierra, 
vuelos a la Luna, despliegue de bases lunares y viajes interplanetarios. Atrapados en el espacio, por su parte, fue el primer filme en utilizar el logo de la NASA y mostraba tecnología en curso de los programas norteamericano y soviético mediante la inclusión de naves Apolo y Vosjod. Concluido el programa lunar, se produjo un paréntesis de catorce años en el género con la excepción de Capricornio 1 (Capricorn One, Peter Hyams, 1977), filme de trama conspiratoria influido por el caso Watergate.

En 1983, Philip Kaufman realiza Elegidos para la gloria (The Right Stuff, Philip Kaufman), una retrospectiva nostálgica sobre los astronautas del proyecto Mercury. Basado en la novela homónima de Tom Wolfe, la película configuró los referentes narrativos del género espacial según Hollywood: doble dimensión familiar y aventurera del arquetipo del astronauta, caracterizado como audaz piloto, ciudadano ejemplar y hombre familiar; conexión patriótica entre el hogar doméstico y el hogar nacional; denuncia del estereotipo femenino promovido por la política de comunicación de la NASA, que instrumentalizaba a las esposas como refuerzo de la estabilidad del programa; y, finalmente, incorporación de los referentes narrativos de la aventura clásica (Sánchez-Escalonilla, 2019).

En lo que respecta al imaginario soviético, el cine y la propaganda representaron las misiones de los cosmonautas Yuri Gagarin, Valentina Tereshkova o Alexei Leonov como triunfos del proletariado, por lo que se evitó la singularización de los héroes y heroínas espaciales mediante ficciones cinematográficas y se optó por la promoción de documentales de calidad. El carácter colectivista de la empresa cósmica suponía el anonimato de cosmonautas e ingenieros, al tiempo que se envolvía en el misterio las propias misiones del programa. Como explica Millard:

La NASA era una agencia civil que hacía público todo cuanto realizaba. Estaba sujeta a una cobertura televisiva completa. Pero esto no existía en el lado soviético, sometido a una estructura cuasi-militar. Muchos de los acontecimientos no se revelaban por entonces, como es el caso del programa lunar ruso (House, 2016). 
Los primeros años del programa liderado por Koroliov coincidieron con la aparición de dos documentales de relieve. El primero, Camino a las estrellas (Doroga $k$ zvezdam, Pavel Kushantsev, 1957), especulaba con el futuro de la humanidad en el cosmos y aventuraba el desarrollo de una estación espacial y de una primera misión lunar. Su estreno se produjo durante el lanzamiento del Sputnik, logro que obligó a introducir en el filme metraje adicional. El segundo documental, también dirigido por Kushantsev, llevaba por título Luna y se estrenó en 1965, el mismo año en que el Leonov realizaba el primer paseo espacial de la historia. Tres años después, el director realiza Marte (Mars): un nuevo documental que, a diferencia de los anteriores, no coincidiría con un éxito ruso en el espacio: en las Navidades de 1968, el Apolo 8 realizó un vuelo de circunnavegación lunar que, de hecho, supuso el fracaso definitivo de la Unión Soviética en la carrera espacial.

Durante la década de los 6o, el cine y los medios de masas habían promovido en la población un entusiasmo tecnológico que Raspopina define como "culto a la ciencia”, y que marcó el imaginario de una época. Como describe la experta:

Los parques infantiles se diseñaban en forma de cohetes, los muros de los colegios y guarderías se decoraban con papel de naves y estrellas. Las casas se construían para que pareciesen naves espaciales, estaciones lunares y platillos volantes: hasta hoy, los expertos se refieren a los años que discurren entre los 60 y los 80 como el "período cósmico" de la arquitectura soviética (Raspopina, 2016).

\section{La nostalgia del sueño espacial. Recuperación de la iconografía tradicional}

Cerrado el período de fragua de los imaginarios espaciales de cada potencia, durante los años 90 se produce un letargo en el programa ruso que dejaría a la NASA la iniciativa de las misiones espaciales durante dos décadas. En este segundo período, el cine de viajes espaciales de Hollywood experimenta una edad de oro iniciada con Apolo 13 (Ron Howard 1995), estrenada para conmemorar el $25^{\circ}$ aniversario de la misión lunar, título al que seguirían 
otros como Contact (Robert Zemeckis 1997), Deep Impact (Mimi Leder 1998), Misión a Marte (Mission to Mars, Brian De Palma 2000) y Space Cowboys (Clint Eastwood 2000). Esta proliferación de títulos se debió en parte a la creación de un departamento de comunicación de la NASA (Dawson 2000) que, al tiempo que promocionaba su logo, prestaba asesoramiento a las productoras.

Si bien el cine ruso no experimentó un auge similar, la agencia Roscomos creada en 1992 era por entonces el segundo socio más destacado de la Estación Espacial Internacional, operativa desde octubre de 2000. Por otro lado, la mayor parte de los países de la AEE participaban en las misiones de la estación orbital. El sueño espacial europeo comenzaba a hacerse realidad con la participación de sus propios astronautas, si bien la agencia carecía de medios y financiación para desarrollar un verdadero sueño alternativo a la NASA o a Roscosmos.

\subsection{Renacimiento espacial como reacción al apocalipsis del American Dream}

Durante la primera década del siglo XXI, el género de viajes espaciales desaparece del panorama fílmico estadounidense, ruso y europeo. En lo que respecta a Estados Unidos, la crisis originada por los atentados del 11S, la guerra de Irak y la recesión económica de 2007 supuso un golpe al espíritu del sueño americano y, por ende, del sueño espacial.

En este período proliferan los espectáculos fílmicos de destrucción como expresión de una cultura del apocalipsis, afianzada mediante géneros de acción como el thriller, la fantasía o el cine bélico (Dixon, 2016; Quart y Auster, 2011). Por contra, el género de exploración espacial no conoció nuevas producciones hasta el estreno de Gravity (Alfonso Cuarón 2013). Este filme propició una segunda edad dorada para el cine de viajes espaciales, género que contribuyó a la renovación del concepto de sueño americano tras una de sus peores crisis. Al filme de Cuarón seguirían Interstellar (Christopher Nolan, 2014), Marte (The Martian, Ridley Scott 2015), Tomorrowland (Brad Bird 2015), Figuras ocultas (Hidden Figures, Theodor 
Melphi, 2016), First Man (Damien Chazelle, 2018) y Ad Astra (James Grey, 2019).

En contraste con las narrativas del desastre, estas nuevas producciones promovieron en la audiencia un espíritu optimista de reconstrucción y refundación en sintonía con los aspectos emergentes del nuevo sueño americano del siglo XXI, que primaba los aspectos metafísicos por encima de los beneficios materiales, replanteaba los valores sociales y humanos como garantía para las nuevas generaciones (Kimmage 2011), y apostaba por un verdadero equilibrio entre hombres y mujeres a la hora de acceder a la prosperidad (Hanson y White (2011).

La nostalgia del espíritu espacial, tal como fue concebido en Estados Unidos en los años 50 y 60, es otro factor presente en los títulos destacados. Se trata de un aspecto que apela a los vínculos intergeneracionales, habituales en toda reflexión histórica sobre la identidad nacional, que ha inspirado tramas de reconstrucción del pasado - caso de los biopics Figuras ocultas y First Man- y relatos de ciencia-ficción futurista - caso de Interstellar y Marte-. Así, los directores Alfonso Cuarón y Brad Bird o el guionista Jonathan Nolan -autor del guion de Interstellar junto a su hermano Christopher- han declarado su deseo de evocar el espíritu de aventura que prendió en la sociedad en los tiempos de la carrera espacial (Weintraub, 2014). Al cabo de una etapa considerada por Time como la "década del infierno"4, los tres directores abordaban sus aventuras espaciales con la mirada puesta en su infancia y con actitud optimista, uno de los rasgos regeneradores del American dream (Kimmage, 2011).

\subsection{El espacio como reivindicación nacionalista rusa}

A diferencia de Hollywood, en la primera década del siglo se estrenaron dos filmes espaciales de relieve, si bien ofrecían visión crítica con el programa soviético: se trata del drama El sueño espacial (Kosmos kak predchuvstvie, Alexei Uchitel, 2005) y el falso documental Los primeros en la Luna (Pervye na Lune, Alexie Fedorchenko, 2005). Como advierte Lewis, "mientras El

\footnotetext{
4 "The Decade from Hell. And why the next one will be better", Time, 7 de diciembre de 2009, vol. 174 núm. 22.
} 
sueño espacial parodia el potencial desaprovechado del programa espacial, frustrado por un sistema corrupto, Los primeros en la Luna retratan a los cosmonautas como víctimas trágicas del Estado" (2008, p. 267). Sin embargo, este tono crítico desaparece a partir de 2013 pues, como también ocurre en Hollywood, el cine ruso experimenta un fenómeno de recuperación nostálgica del sueño espacial. Así sucede en Gagarin: el primero en el espacio (Gagarin. Pervyy $v$ kosmose, Pavel Parkhomenko 2013), Spacewalker (Vremia pervyj, Dimitri Kiselev 2017) y Salyut 7: héroes en el espacio (Salyut 7, Klim Shipenko 2017), biopics en los que se aprecia un marcado componente nacionalista.

Según Maurer, este fenómeno surgió dentro del contexto de renovación patriótica promovido en los medios desde instancia políticas, en especial tras la constitución del comité para la celebración del $50^{\circ}$ aniversario de Gagarin en el espacio, que concluyó en la declaración de 2011 como "Año de los Cosmonautas Rusos". En 2009, el presidente Vladimir Putin se dirigió a este comité para expresar su preocupación sobre lo que consideraba como una "falsificación de la historia" ante el olvido de la gesta rusa del primer hombre en el espacio: el aniversario sería una oportunidad para señalar "ante el público del mundo el papel crucial de Rusia en la conquista del cosmos" (Maurer 2011, p. 1).

El filme sobre Yuri Gagarin llegó a las pantallas rusas en 2013, promovido con fondos estatales. Gagarin: el primero en el espacio recibió críticas por mostrar lo que se consideraba como un retrato idealizado de su protagonista, de acuerdo con el patrón épico de la era Kruschev (Chilton, 2013). Según el semanario ruso Ogonyok, "el cine soviético trató a Gagarin como a un dios, pues retratarlo se consideraba un pecado, pero para el cine post-soviético [Gagarin] es como una metáfora de la vida soviética" (Malpas, 2013).

La tendencia patriótica del cine espacial ruso se mantuvo en Spacewalker y Salyut 7, aparecidos en 2017 y más afines a la pauta marcada en el Hollywood de los 80 con Elegidos para la gloria. En Spacewalker, Kiselev recreaba el primer paseo espacial de Leonov y enmarcaba el hito en la competición con la NASA. Salyut 7, por su parte, relataba con esmerados 
efectos visuales la lucha de dos cosmonautas por reparar la estación orbital rusa durante una trama de supervivencia que recordaba el tono de Apolo 13. El filme de Shipenko mostraba la hazaña como una demostración de fuerza ante la NASA y la administración de Reagan, tal como se refleja en el plano final en que la admirada tripulación del transbordador saluda a los cosmonautas a través de la carlinga. Por otro lado, el filme no ocultaba su postura crítica con el estamento militar que supervisaba el programa espacial en 1985 .

El aspecto nostálgico de los filmes rusos presenta un componente de reafirmación nacionalista que recuerda los primeros biopics estadounidenses del género. Entre 2013 y 2019, el arquetipo del astronauta de Hollywood ya había evolucionado hacia un patrón dramático alejado del protagonismo masculino, las misiones se comparten con otras potencias espaciales y se llega a adoptar una postura crítica con las decisiones de la agencia espacial, a menudo influidas por criterios políticos o propagandísticos (SánchezEscalonilla, 2018). Por contra, el arquetipo idealizado del cosmonauta en los filmes rusos posteriores a 2013 se ajusta más bien al icono de la Guerra Fría, según la descripción de Gerovitch:

El mito del cosmonauta, un héroe perfecto que conquista el espacio exterior con tecnología infalible, se alimentaba - y al mismo tiempo sustentaba- de un mito político aún más grande: el de la Unión Soviética como una superpotencia que producía héroes perfectos y creaba una tecnología infalible (Gerovitch, 2011, p. 463).

\section{Próxima y la renovación de arquetipos espaciales}

En 2019, año en que se celebró el $50^{\circ}$ aniversario de la llegada a la Luna, se produjo el estreno de Próxima: un filme que irrumpía en la categoría de viajes espaciales como una alternativa europea a la tradicional polaridad de los sueños americano y ruso. Se trataba de la primera incursión de la realizadora en el género, tras el drama histórico Augustine (2012) y el thriller Disorder: El protector (Maryland, 2015). Winocour también había firmado el guion de Mustang (2015) junto a su directora, la turco-francesa Deniz 
Gamze Ergüven, en una colaboración que denunciaba la situación de atraso social sufrida por las jóvenes turcas.

Las dos realizadoras se habían conocido mientras cursaban el programa Cinéfondation del Festival de Cine de Cannes, orientado a la promoción de jóvenes que abordan su primer o segundo largometraje, y durante la experiencia - eran las dos únicas mujeres- comprobaron que les unían los mismos intereses vitales y narrativos (Fesenthal, 2015). Pocos años más tarde, Winocour inició la preproducción de un nuevo filme sobre un relato de superación nuevamente protagonizado por una mujer, esta vez con el espacio como escenario. Próxima narra la historia de Sarah Loreau (Eva Green), una astronauta francesa que se prepara en el centro de la Agencia Espacial Europea de Colonia para viajar a la Estación Espacial Internacional, donde permanecerá un año entero como parte de los preparativos de una futura misión a Marte.

El concepto de sueño espacial se presenta, de entrada, como un objetivo particular que encuentra su primer obstáculo en el ámbito personal. Sarah siempre ha deseado viajar a las estrellas, pero la inminente separación de su hija pequeña, Stella, ha levantado una barrera entre las dos. La presión de los simulacros que realiza en la Ciudad de las Estrellas, en las cercanías de Moscú, suponen un sufrimiento menor comparado con el peligro de perder el afecto de Stella. Esta tensión entre la vida profesional y la vida personal, ya tratada en Augustine y Disorder, expande el conflicto a proporciones cósmicas. En opinión de Mintzer,

[Este escenario] abarca las increíbles tareas que se demandan de un astronauta mientras se preparan para una misión, y muestra las hazañas sobrehumanas que se requieren de las personas, de una mujer en este caso específico, hazañas sin embargo demasiado humanas. Este tema también recuerda el reciente biopic sobre Neil Armstrong First Man, y de hecho esta película podría titularse First Woman con todo orgullo y eficacia (Mintzer, 2019). 
En segundo lugar, el filme plantea como conflicto dramático las dificultades de la mujer astronauta para acceder a la realización del sueño espacial en misiones tradicionalmente protagonizadas por hombres. Finalmente, Próxima ofrece un tercer nivel de conflicto al introducir un tripulante europeo como protagonista junto a los miembros estadounidense y ruso, secundarios del drama.

Mediante este triple conflicto, Winocour presenta una hibridación superadora de los imaginarios tradicionales con una apuesta narrativa por la vía intermedia de un sueño espacial europeo, carente de iconografía fílmica. Por este motivo, el personaje de Sarah irrumpe con mayor rotundidad en un escenario diegético donde el diseño de producción y la fidelidad tecnológica favorecen la verosimilitud de la historia. Como una especie de intrusión en el arquetipo clásico, el miembro europeo se une a la tripulación dentro del módulo de mando de la nave Soyuz, junto a los representantes de la NASA y de Roscosmos. Examinemos con más detalle esta colisión con los sueños espaciales previos, a través de esta reinvención del arquetipo de astronauta que proporciona Winocour.

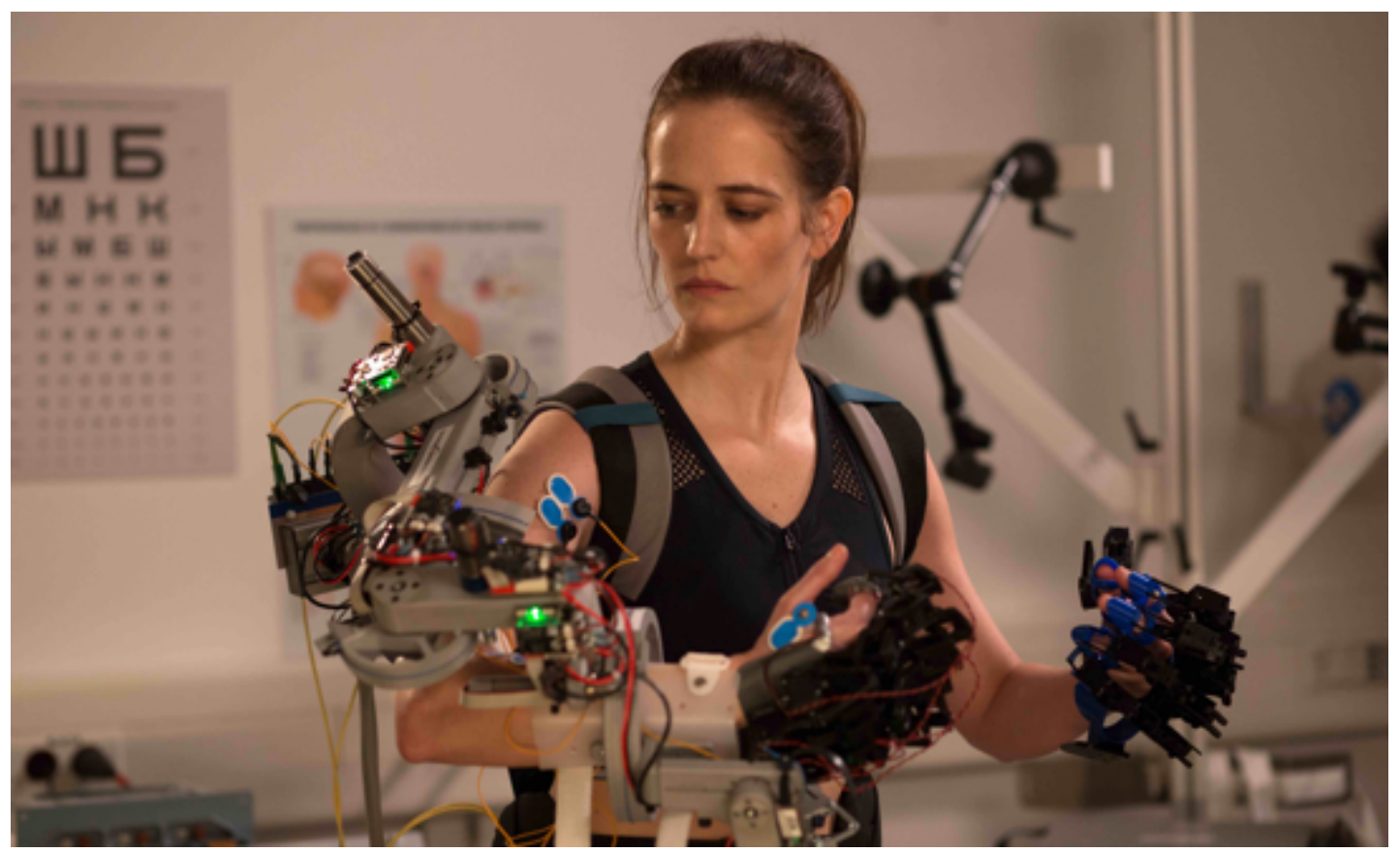

F1 Próxima, Alice Winocour, 2019 C Dharamsala / Darius 


\subsection{Contraste con el sueño espacial americano}

Sarah es protagonista, mujer, madre y europea. Se trata de cuatro aspectos que, de entrada, alejan al personaje de la iconografía del sueño espacial estadounidense $\mathrm{y}$, por extensión, del cliché de astronauta divulgado por la filmografía de Hollywood. Durante los entrenamientos, Sarah tiene ocasión de tratar a su compañero de tripulación de la NASA, Mike Shannon (Matt Dillon). Este secundario se plantea, de entrada, como la némesis de Sarah pues se ajusta al arquetipo tradicional creado en Elegidos para la gloria.

Mike aglutina a los astronautas masculinos representados en el cine de los 80 y 90: herederos del prototipo popular creado por los programas Mercury, Gemini y Apolo, todos ellos mostraban a hombres de acción que dejaban a sus esposas la responsabilidad total sobre sus hijos mientras realizaban sus periplos cósmicos. Frente a Sarah, el personaje estadounidense porta una aureola de héroe forjada durante décadas de sueño espacial, y esto le lleva a adoptar una actitud de cierta prepotencia durante los entrenamientos. McCurdy se refiere así al origen de este cliché:

En el amanecer de la era espacial, las obras de la imaginación reforzaron los estereotipos sexuales. Los cohetes y las naves espaciales se presentaban como juguetes caros, pero solo aptos para hombres o muchachos. Las mujeres se representaban como tecnológicamente torpes e incapaces de comprender la ingeniería tecnológica, por no hablar de su presunta incapacidad para mandar sobre hombres o pilotar una nave espacial (McCurdy, 2011, p. 295).

Por otro lado, durante los programas pioneros de la NASA, la agencia promovió una campaña mediática de sus astronautas fundamentalmente a través de la revista Life-donde la imagen de las esposas se empleaba para reforzar la aceptación social de los héroes espaciales. Mientras se desarrollaba el programa lunar, John Sturges 
ya señaló esta manipulación en el filme Atrapados en el espacio, denuncia que reaparece en Apolo 13 y Elegidos para la gloria. En la novela homónima de este último título, Tom Wolfe escribía: "La esposa iniciaba su matrimonio (con su marido y con el ejército) haciendo ciertos pequeños sacrificios. Sabía que la paga sería ruin y miserable. Tendrían que trasladarse con frecuencia y vivir en casas deprimentes y destartaladas" (2010, pp. 128-129).

En la segunda década del siglo, el arquetipo espacial femenino evoluciona en Hollywood desde la condición secundaria de esposa relegada al hogar y al cuidado de los hijos, hasta la condición protagonista de científica y viajera estelar. La doctora Stone de Gravity constituye un hito en el nuevo cliché, al que seguirían la doctora Brand en Interstellar, la comandante Lewis a bordo de la estación Ares en Marte, y las científicas afroamericanas de Figuras ocultas, filme que ponía en valor su trabajo silenciado durante el programa Mercury. Por otro lado, First Man situaba el papel de Jane Armstrong a la altura épica de su esposo, protagonista de la gesta lunar.

El conflicto tradicional entre el hogar familiar y el espacio - nota característica de la filmografía espacial estadounidense-, alcanza su cota máxima gracias al personaje de Sarah Loreau. En efecto, la mujer astronauta aúna las figuras de la madre y de la científica, personajes pasivos en tierra, y las eleva a un terreno dramático incógnito para el cine hasta encontrar una nueva categoría. Sin embargo, este súbito acceso al sueño espacial entraña el conflicto interno de la separación de una hija pequeña. A este respecto, la directora de Próxima recordaba:

Una mujer de la NASA me dijo que el mejor entrenamiento que había tenido para convertirse en astronauta había sido ser madre, porque una madre está inmersa constantemente en la multitarea. Una entrenadora de la 
AEE me dijo que los astronautas varones hablan con mucho orgullo de sus hijos, mientras que las mujeres astronautas tienden a ocultar el hecho de que son madres, como si temieran perder credibilidad. Prevalece esa idea, un puro constructo social, de que un hijo es principalmente responsabilidad de su madre (Winocour, 2019, p. 3).

La idea de separación, tratada en el género durante décadas para humanizar al personaje masculino del astronauta, adquiere en Próxima una perspectiva antropológica más audaz en términos dramáticos, pues permite a su directora ir más allá de las estrategias emocionales y abordar un problema de desigualdad. El relato parte de la idea de que una madre resulta insustituible en la Tierra una vez abandona el planeta y se somete a la ingravidez, a la liberación de la atracción física. De manera simbólica, Mike insta a Sarah a "cortar el cable" durante uno de sus entrenamientos, estableciendo así una colisión alegórica entre su doble misión como madre y como astronauta.

Esta tensión se refleja, por otro lado, en el personaje secundario de Thomas, su ex-marido y padre de Stella, quien resulta incapaz de cubrir la carencia afectiva de la niña. Por otro lado, la posición del personaje en este conflicto se plasma en su condición de astrofísico de la Agencia Espacial Europea: como se deduce de su implicación en misiones no tripuladas a Marte, su vivencia del sueño espacial no incluye ningún riesgo de tipo físico o familiar. Alice Winocour redime en el guion el sentimiento de culpabilidad de Sarah ante su deseo de realizar su sueño espacial:

He querido mostrar sobre todo lo difícil que se hace para una mujer el tipo de separación que describo, porque ella es la que suele ocuparse más intensamente de los hijos y, por tanto, desarrolla más fácilmente un sentido de culpabilidad cuando no puede hacerlo. La sociedad nos dice esto pero, como se ve en el filme, un padre también puede hacerlo, aprender a desenvolverse en este tipo de situaciones. Acaba ayudando a la 
madre y a la niña, a liberarse. La niña debe liberarse del sueño del espacio de su madre (Aresté, 2019).

La mencionada alegoría de "cortar el cable" se emplea también en Gravity, filme que guarda cierta similitud con Próxima. Sarah es, en efecto, la primera mujer astronauta europea de la historia del cine con un papel protagonista mientras que otra Sarah, la doctora Stone de Gravity, interpreta a la primera mujer astronauta estadounidense con un papel principal en una producción de Hollywood. En los dos filmes, la gravedad física y la separación de sus hijas - definitiva en el caso de Stone- oprimen a las dos astronautas. Y para las dos, el sufrimiento de la misión les permite afrontar una purificación de sus identidades como madres, como aventureras espaciales y como representantes de la humanidad en un contexto cósmico marcado por las tensiones internacionales.

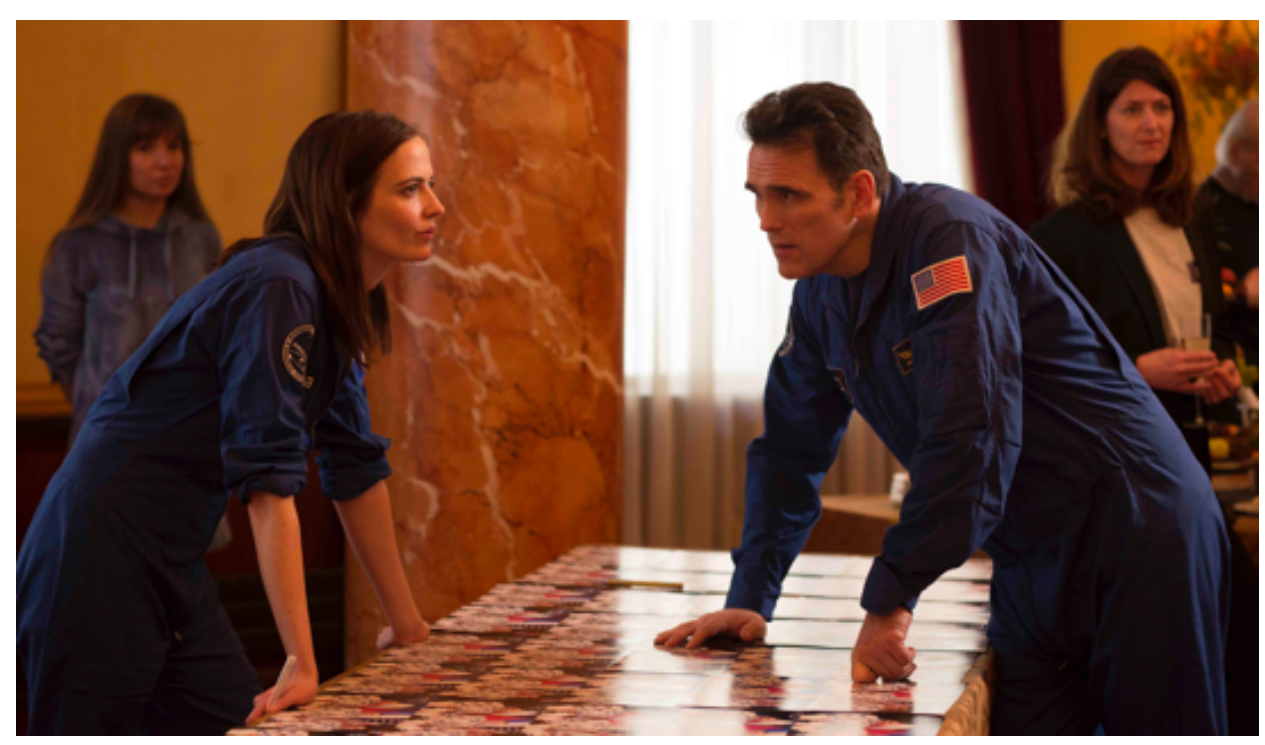

F2 Próxima, Alice Winocour, 2019 (C) Dharamsala / Darius

\subsection{Contraste con el sueño espacial ruso}

Anton Ochievski (Alexei Fateev) es el representante del sueño espacial ruso a bordo del Soyuz, junto a Sarah y a Mike. Aunque se trata de un personaje algo más desdibujado que sus compañeros de tripulación, su presencia condensa la tradición de los cosmonautas 
junto al sueño estadounidense y al incipiente sueño europeo que plantea el filme. El proyecto de Próxima se gestó en la estela de celebraciones de los aniversarios que, por incentivo estatal, rememoraban las hazañas de la agencia rusa a través de filmes como Salyut 7 o Gagarin. Sin embargo, en cuanto representante de la agencia europea, Sarah no tiene nada que celebrar en el espacio. Su presencia a bordo de la nave no entraña interés nacionalista, ni puede suponer nostalgia alguna por gestas del pasado. En cuanto europea, se trata de un personaje completamente pionero.

Si el contraste entre Mike y Sarah enfatiza la feminidad de la protagonista, el contraste con Anton pone de relieve la singularidad de la astronauta como representante de un sueño europeo por realizar. La europeidad de Sarah no solo procede de su pertenencia a la AEE, o de su condición de madre francesa que comparte una hija con un científico alemán. Los astronautas europeos a bordo de las naves Soyuz aglutinan el sueño espacial de veintidós estados miembros, en un esfuerzo común que, a diferencia de las potencias de la Guerra Fría, ha nacido desvinculado de pretensiones geopolíticas. Por otro lado, el cine europeo tampoco cuenta con una tradición iconográfica con la que Sarah pueda identificarse, o que proporcione a Winocour la narrativa de un imaginario nostálgico como el que, a partir de 2013, ha servido de apoyo a Hollywood y a la industria rusa.

Aunque buena parte de la acción transcurre en la Ciudad de las Estrellas de Moscú y en el cosmódromo de Baikonur, la protagonista de Próxima puede considerarse un prototipo europeo de astronauta que trasciende las barreras nacionalistas y los imaginarios del cine espacial. "Mi objetivo era hacer una película que fuera europea", confirma Winocour (2019, p. 2). La directora también deseaba demostrar que la cooperación internacional funciona mejor en el 
espacio que en el ámbito geopolítico: el carácter internacional de la propia producción así lo demostraba.

La condición de Sarah como mujer y madre astronauta supone también un contraste con la filmografía rusa. Durante la era soviética, el ya comentado carácter colectivo de los protagonistas espaciales conllevó el anonimato de las mujeres en los filmes documentales. Por otro lado, los biopics posteriores a 2013 Salyut 7, Gagarin y Spacewalker abundaban en el arquetipo femenino de las esposas relegadas a papeles secundarios. La única excepción corresponde a la breve aparición en Salyut 7 de la cosmonauta Svetlana Savítskaya, primera mujer que realizó un paseo espacial. Por otro lado, en la tendencia nostálgica hacia la carrera espacial resulta patente la ausencia de un filme sobre Valentina Tereshkova, primera mujer en el espacio.

Respecto al sueño espacial ruso, Próxima contrasta como producción desprovista de contenidos nacionalistas o nostálgicos que, al mismo tiempo, ha rescatado el heroísmo de las aventureras espaciales. A propósito del predominio masculino en el imaginario espacial contemporáneo, la directora se refiere así a las escenas de Próxima durante los entrenamientos en la Ciudad de las Estrellas:

Puede parecer que esas escenas son caricaturescas, pero siguen siendo menos fuertes que las experiencias reales de las que me hablaron las mujeres astronautas. Se trata de un entorno masculino, dirigido por y para hombres. Por ejemplo, los trajes espaciales llevan el peso en los hombros, porque los hombres tienen los hombros fuertes, mientras que las mujeres tienen la fuerza en las caderas. Las mujeres tienen que esforzarse el doble para conseguir entrar en ese mundo de hombres, pero no deben hacer notar su presencia. La película rinde homenaje a las mujeres que tienen que conciliar todo esto, y evidentemente en el negocio de la exploración del espacio esto se ve exacerbado (Winocour 2019, p. 3). 
A la hora de visualizar la aventura de Sarah, el filme abandona pronto los escenarios de la Agencia Espacial Europea en Colonia para situarse en la Ciudad de las Estrellas de Moscú y el cosmódromo de Kazajistán. Estas dos localizaciones guardan un poderoso vínculo iconográfico con el sueño espacial ruso, al tiempo que se asocian con entornos de alta seguridad y carácter militar. La presencia de la astronauta europea en estos escenarios coincide con el agravamiento de su conflicto interior, marcado por la separación de su hija Stella. Por este motivo, el único sentimiento de nostalgia que late en Sarah no está vinculado a las gestas astronáuticas del pasado sino al recuerdo de su propia hija.

Según la directora, las fases del guion discurren en paralelo con la separación por etapas del cohete Soyuz, que a su vez reflejan el distanciamiento entre madre e hija, incluida la fase de la llamada "separación umbilical". Esta idea surgió durante la estancia de Winocour en el centro espacial de Colonia, donde se le ocurrió que "la relación madre-hija es comparable a la relación que la humanidad mantiene con la Tierra, y que la separación de la Tierra experimentada por el astronauta sonaba como la separación de Sarah y su hija” (European Space Agency, 2019).

En este contexto, la etimología del término nostalgia (“dolor del hogar" en griego antiguo) resulta más adecuada cuando se aplica al ámbito familiar del personaje y no tanto al ámbito nacional o global. Durante los entrenamientos, la presión de los simulacros de emergencias en la Ciudad de las Estrellas, en las cercanías de Moscú, suponen a Sarah un sufrimiento menor comparado con el peligro real de perder el afecto de Stella. Como advierte a Sarah una veterana rusa de los programas espaciales, la dificultad no consiste en el lanzamiento ni en la supervivencia allá arriba: "Lo más duro es regresar, cuando compruebas que la vida ha seguido aquí abajo sin ti”. 


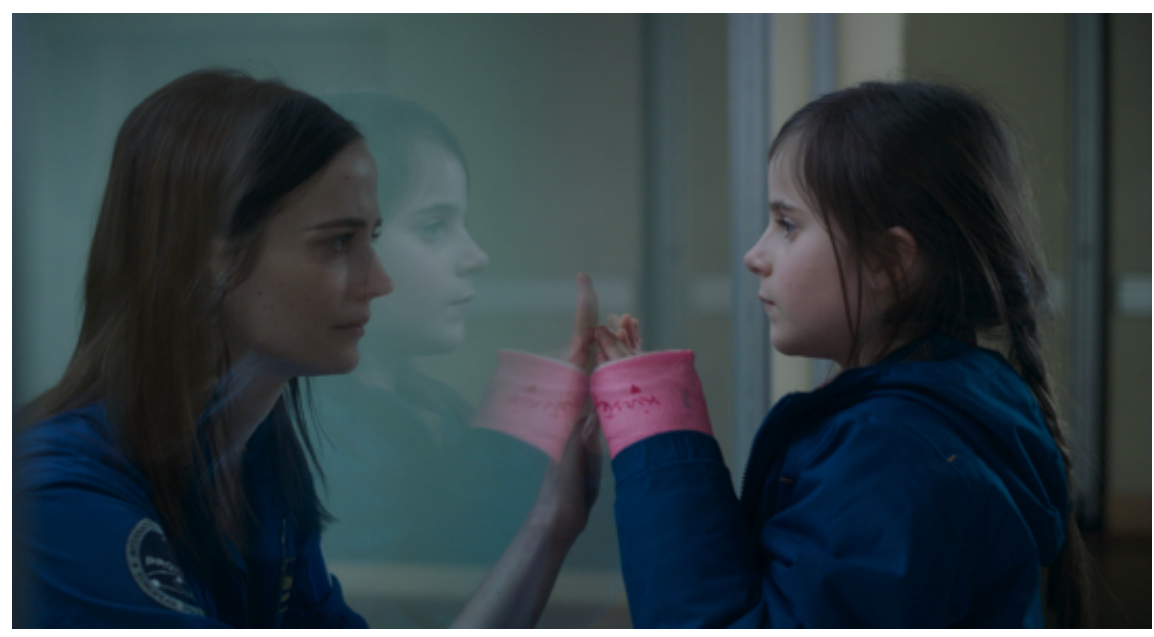

F3 Próxima, Alice Winocour, 2019 (c) Dharamsala / Darius

\section{Conclusiones}

Alice Winocour cierra Próxima con un homenaje a las madres que han viajado al espacio, cuyos nombres y rostros se incluyen en los créditos finales. Entre ellas se encuentran mujeres astronautas estadounidenses, rusas y europeas. De manera simbólica, el filme hace patente los rasgos con que Rifkin sintetiza el concepto de sueño europeo y su propósito de proporcionar a la humanidad una conciencia común, en una sociedad interconectada y globalizada (2004). Así, el filme refleja un sueño espacial emergente que supera la dicotomía de bloques entre American Dream y ethos soviético, mediante una hibridación de sus propios imaginarios.

En una década marcada por la nostalgia de las gestas espaciales, el filme plantea de modo implícito una renovación iconográfica del género en cuatro dimensiones: espacial, socionarrativa, geopolítica y épica. Todos ellos se integran en el personaje de Sarah, primera mujer astronauta europea con papel protagonista en una producción asimismo europea. De este modo, el personaje se convierte en un prototipo heroico femenino capaz de superar las barreras que obstaculizan el viaje al espacio como reto personal: la separación de su hija, la presión causada por el enfoque masculino de su profesión y la débil tradición espacial europea. Winocour parte del imaginario tradicional presente en la filmografía espacial rusa y 
estadounidense para mostrar el aspecto de un sueño alternativo, mediante una ficción que contiene en su estilo elementos del género documental y que, a la postre, podría considerarse un biopic colectivo.

Por otro lado, la condición europea de Sarah se aprecia en el filme como una tercera vía que trasciende las rivalidades entre potencias y pone en alza los nuevos valores del sueño espacial, marcado por el globalismo y la cooperación entre naciones y no por la reivindicación de gestas nacionales. Esta visión de Winocour se encuentra más cercana al sueño espacial ecuménico presente en producciones estadounidenses de la década como Gravity o Marte, en cuyas tramas se muestra una labor integrada entre diferentes agencias como la Roscosmos rusa, la NASA, la ESA y la Administración Espacial Nacional China, al tiempo que se advierte sobre las consecuencias fatales de una concepción geopolítica del espacio.

Según propone Dixon, el cine de esta y de las décadas sucesivas debe contribuir a superar las que denomina "preocupaciones apocalípticas" como el calentamiento global, la hambruna masiva, las olas de migrantes empobrecidos por la guerra o la proliferación popular de armas: “Todas estas cosas apuntan hacia una sociedad en crisis, una crisis también para la maquinaria cinematográfica que crea los sueños que esta sociedad necesita, tan desesperadamente, si desea hacerse a sí misma” (Dixon 2016: 1-2). De acuerdo con la estimación del experto, Próxima podría considerarse como uno de esos sueños necesarios.

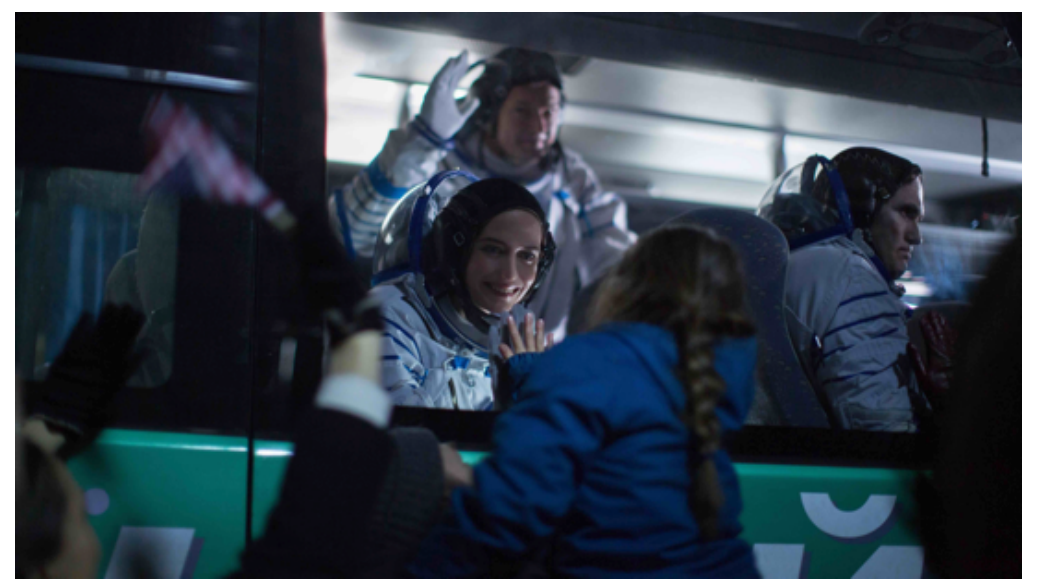

F4 Próxima, Alice Winocour, 2019 @ Dharamsala / Darius 


\section{Referencias bibliográficas}

Abeelen, Luc van der (2016). Spaceplane HERMES: Europe's Dream of Independent Manned Spaceflight. Hilversum: Springer.

Adams, James Truslow (2017). The Epic of America. Abingdon, Oxon: Routledge. $1^{\text {a }}$ ed. 1931, Boston: Little, Brown.

Allen, M. (2009). Live from the Moon. Film, Television and the Space Race. Nueva Yok: Taurus.

Andrews, J. (2011). Getting Ready for Krushchev's Sputnik. Russian Popular Culture and National Markets at the Dawn of the Space Age. En Andrews, J. y Siddiqi (Eds.), Into the Cosmos: Space Exploration and Soviet Culture (pp. 28-44). Pittsburgh: University of Pittsburgh Press.

Andrews, J. y Siddiqi, A. (2011). Space Exploration in the Soviet Context. En J. Andrews, y A. Siddiqi (Eds.), Into the Cosmos: Space Exploration and Soviet Culture, (pp. 1-14). Pittsburgh: University of Pittsburgh Press.

Aresté, J.M. (2019, 12 de diciembre). Alice Winocour, muy próxima. DeCine21. Diario digital de cine $y$ series. https://decine21.com/entrevistas/119119-alice-winocour-muyPróxima-ser-astronauta-es-una-experiencia-de-fragilidad

Chilton, Martin (2013, 19 de junio). Yuri Gagarin attracts criticism. The Telegraph. $\quad$ https://www.telegraph.co.uk/culture/film/filmnews/10129255/Yuri-Gagarin-movie-attracts-criticism.html

Collins, Guy (1990). Europe in Space. Nueva York: Palgrave Macmillan.

Dawson, J. (2000, 7 de abril). Dread Planet. Guardian, p. 10.

Dixon, W. (2016). (2016). Hollywood in Crisis or: The Collapse of the Real. Nueva York: Palgrave Macmillan.

Fesenthal, J. (2015, 13 de noviembre) Deniz Gamze Ergüven on Her Stunning New Movie, Mustang.

Vogue.

https://www.vogue.com/article/mustang-deniz-gamze-erguveninterview

Gerovitch, S. (2011). Why Are We Telling Lies? The Creation of Soviet Space History Myths. The Russian Review 70(3), 463.

Hanson, S. y White, J. (Eds.) (2011). The American Dream in the $21^{\text {st }}$ Century. Filadelfia: Temple University Press.

Harrod, M., M. Liz y A. Timoshkina (Eds.) (2015). The Europeanness of European Cinema. Identity, Meaning, Globalization. Londres: I.B. Tauris.

House, A. (2016). Exhibition Space. How the greatest show on Earth is counting-down to lift-off. Space Soviet Dreams. The Calvert Journal https://www.calvertjournal.com/features/show/4654/spaceinterview-cosmonauts-science-museum-doug-millard-alexandrasmirnova 
Kimmage, M. (2011). The Politics of the American Dream, 1980 to 2008. En S. Hanson y J. White (Eds.). The American Dream in the $21^{\text {st }}$ Century (167-200). Filadelfia: Temple University Press.

Laqueur, Walter (2012). After the Fall: The End of the European Dream and the Decline of a Continent. Nueva York: St Martin's Press.

Lewis, C. (2008). From the Cradle to the the Grave. Cosmonaut Nostalgia in Soviet and Post-Soviet Film. En S. Dick (Ed.), Remembering the Space Age (253-270). Washington DC: NASA, History Division.

Malpas, A. (2013, 16 de junio). First Gagarin film turns Soviet idol into new Russian hero. Phys. https://phys.org/news/2013-06-gagarin-sovietidol-russian-hero.html

Maurer, E. et al. (2011). Soviet Space Culture. Cosmic Enthusiasm in Socialist Societies. Nueva York: Palgrave Macmillan.

McCurdy, Howard E. (2011). Space and the American Imagination. Baltimore: The John Hopkins University Press.

McDougall, Walter A. (1985). ...the Heavens and the Earth: A Political History of the Space Age. Nueva York: Basic Books.

Migration Data Portal (2015). https://migrationdataportal.org/data?t=null

Mintzer, J. (2019, 9 de junio). Próxima. Toronto International Film Festival. Hollywood https://www.hollywoodreporter.com/review/Próxima-review$\underline{1237508}$

Park, Jeanne (30 de septiembre de 2015) Europe's Migration Crisis, Council of Foreign Relations.

https://www.cfr.org/backgrounder/europes-migration-crisis

Quart y Auster (2011). American Film and Society since 1945. Santa Bárbara, California: Praeger.

Raspopina, S. (2016). Cult of the cosmic: How space travel became the unofficial religion of the USSR. Space Soviet Dreams. The Calvert Journal. https://www.calvertjournal.com/features/show/4645/spacepropaganda-posters-songs-soviet-religion

Rifkin, J. (2004). The European Dream. How Europe's Vision of the Future is Quietly Eclipsing the American Dream. Cambridge: Polity Press.

Samuel, L. (2012). The American Dream. A Cultural History. Nueva York: Siracuse University Press.

Sánchez-Escalonilla, A. (2018). El cine de exploración espacial del siglo XXI: una muestra de renovación narrativa del American Dream. Área Abierta 18(1): 215-231. https://doi.org/10.5209/ARAB.60049

Sánchez-Escalonilla, A. (2019). Planeta Hollywood. American Dream y sueño espacial. Madrid: Encuentro. 
Smith, M. (2014) Rockets and Revolution. A Cultural History of Early Spaceflight. Lincoln: University of Nebraska Press.

Spufford, F. (2010) Red Plenty. Inside the Fifties' Soviet Dream. Londres: Faber and Faber.

Turner, Frederick J. (2014). The Significance of the Frontier in American History. Eastern, Connecticut: Martino Fine Books.

United Space in Europe. (2019, 26 de noviembre). Próxima propulse le spatial européen sur grand écran. European Space Agency. https://www.esa.int/Space in Member_States/France/Próxima_pro pulse_le_spatial_europeen_sur_grand_ecran

Wachhorst, Wyn (2000). The Dream of Spaceflight. Essays on the Near Edge of Infinity. Nueva York: Basic Books.

Weintraub, Steve (2014, 8 de noviembre). Christopher Nolan, Matthew McConaughey, Anne Hathaway, and More Talk Interstellar, the Evolution of the Script, and Grounding the Film with Emotion. Collider http://collider.com/christopher-nolan-interstellar-interview/

Winocour, A. (2019). Próxima. Pressbook. Isabelle Madelaine and Emilie Tisné.

Wolfe, T. (2010). Lo que hay que tener. Elegidos para la gloria. Madrid: Anagrama.

\section{Filmografía}

Bird, B. (2015). Tomorrowland. USA: Walt Disney Pictures.

Chazelle, D. (2018). First Man. USA: Universal.

Cuarón, A. (2013). Gravity. USA: Warner.

De Palma, B. (2000). Mission to Mars. USA: Touchstone.

Eastwood, C. (2000). Space Cowboys. USA: Warner.

Ergüven, D. (2015). Mustang. Turquía/ Francia: CG Cinéma.

Fedorchenko, A. (2005). Pervye na Lune. Rusia: Kinocompanya Strana.

Grey, J. (2019). Ad Astra. USA: Twentieth Century Fox.

Howard, R. (1995). Apollo 13. USA: Universal.

Hyams, P. (1977). Capricorn One. USA: AGF.

Kaufman, P. (1983). The Right Stuff. USA: The Ladd Company.

Kiselev, D. (2017). Vremia pervyj. Rusia: Bazelevs Productions.

Kubrick, S. (1968). 2001: A Space Odyssey. USA: Stanley Kubrick Productions.

Kushantsev, P. (1957). Doroga $k$ zvezdam. URSS: Leningrad Popular Film Studio. 
Kushantsev, P. (1965). Luna. URSS: Leningrad Popular Film Studio.

Kushantsev, P. (1968). Mars. URSS: Leningrad Popular Film Studio.

Leder, M. (1998). Deep Impact. USA: Paramount.

Melphi, T. (2016). Hidden Figures. USA: Fox 2000 Pictures.

Nolan, C. (2014). Interstellar. USA: Paramount.

Parkhomenko, P. (2013). Gagarin. Pervyy $v$ kosmose. Rusia: Kremlin Films.

Scott, R. (2015). The Martian. USA: Twentieth Century Fox.

Shipenko, K. (2017). Salyut 7. Rusia: Globus-Film.

Sturges, J. (1969) Marooned. USA: Columbia.

Uchitel, A. (2005). Kosmos kak predchuvstvie. Rusia: Studio Rock.

Winocour, A. (2012). Augustine. Francia: Dharamsala.

Winocour, A. (2019). Maryland. Francia/Bélgica: Dharamsala.

Winocour, A. (2019). Proxima. Francia/Alemania: Dharamsala.

Zemeckis, R. (1997). Contact. USA: Warner.

Zhuravlyov, V. (1936). Kosmicheskii Reis. URSS: Mosfilm. 